\title{
Ab initio Studies of Atomic Properties and Experimental Behavior of element 119 and Its Lighter Homologs
}

\author{
A. Borschevsky ${ }^{1}$, V. Pershina ${ }^{2}$, E. Eliav ${ }^{3}$, and U. Kaldor ${ }^{3}$ \\ ${ }^{1}$ Helmholtz Institute Mainz, Mainz D-55128, Germany; ${ }^{2}$ GSI, Darmstadt, Germany; ${ }^{3}$ Tel Aviv University, Israel
}

We performed relativistic benchmark calculations of the polarizabilities $(\alpha)$ of element 119 and its lighter homologs, Cs and Ra, and their cations. Besides being of theoretical interest in the context of atomic studies of heavy and superheavy elements, these properties are also important for prediction of adsorption enthalpy $\left(\Delta H_{\text {ads }}\right)$ of the atoms on inert surfaces, which is required to guarantee the transport of the newly produced element from the target chamber to the chemistry set up.

The polarizabilities were calculated using the finite field approach [1]. The energy calculations were performed within the Dirac-Coulomb (DC) Hamiltonian,

$$
H_{\mathrm{DC}}=\sum_{i} h_{D}(i)+\sum_{i<j} 1 / r_{i j}
$$

Here, $h_{D}$ is the one electron Dirac Hamiltonian,

$$
h_{D}(i)=c \boldsymbol{\alpha}_{i} \cdot \mathbf{p}_{i}+c^{2} \beta_{i}+V_{n u c}(i) \text {, }
$$

where $\alpha$ and $\beta$ are the four dimensional Dirac matrices. The nuclear potential $V_{n u c}$ takes into account the finite size of the nucleus, modelled by a Gaussian distribution.

Electron correlation was taken into account at the relativistic coupled cluster level, including single, double, and perturbative triple excitations ( $\operatorname{RCCSD}(\mathrm{T}))$. The uncontracted Faegri basis set [2] was used for the three atoms and extended to convergence with respect to the calculated polarizabilities. The final basis sets were $26 s 23 p 16 d 8 f 4 g$ for Cs, 26s $23 p 18 d 13 f 6 g 2 h$ for $\mathrm{Fr}$, and $29 s 26 p 20 d 15 f 6 g 2 h$ for element 119 . All the calculations were performed using the DIRAC08 computational package [3].

Based on the calculated polarizabilities and other atomic properties and using a physisorption model given by Eq. (6) of Ref. 4, we estimate the $\Delta H_{\text {ads }}$ of group- 1 elements on a Teflon surface. The van der Walls radii $\left(R_{\mathrm{vdW}}\right)$ were determined from a linear correlation between the known $R_{\mathrm{vdW}}$ in Group 1 [5] and the radii of the maximal charge density $\left(R_{\max }\right)$ of the valence $n s$ orbitals [6].

Table 1.Polarizabilities of neutral $(\alpha(\mathrm{M}))$ and singly charged $\left(\alpha\left(\mathrm{M}^{+}\right)\right)$group-1 elements (a.u.), and their $R_{\mathrm{vdW}}(\AA)$ and $\Delta H_{\mathrm{ads}}$ $(\mathrm{kJ} / \mathrm{mol})$ on Telfon. All the values were calculated here, unless reference otherwise.

\begin{tabular}{lllllll}
\hline & $\mathrm{Na}$ & $\mathrm{K}$ & $\mathrm{Rb}$ & $\mathrm{Cs}$ & $\mathrm{Fr}$ & 119 \\
\hline$\alpha(\mathrm{M})$ & $162.7^{\mathrm{a}}$ & $290.6^{\mathrm{a}}$ & $318.8^{\mathrm{a}}$ & 399.0 & 311.5 & 169.7 \\
$\alpha\left(\mathrm{M}^{+}\right)$ & $1.0^{\mathrm{b}}$ & $5.5^{\mathrm{b}}$ & $9.1^{\mathrm{b}}$ & 15.5 & 20.1 & 31.6 \\
$R_{\mathrm{vdW}}$ & $2.27^{\mathrm{c}}$ & $2.75^{\mathrm{c}}$ & 2.90 & 3.16 & 3.09 & 2.78 \\
$-\Delta H_{\text {ads }}$ & 32.7 & 29.3 & 26.6 & 24.6 & 21.2 & 17.6 \\
\hline
\end{tabular}

${ }^{\mathrm{a}}$ Exp., Ref. [7]; ${ }^{\mathrm{b}}$ Theor., Ref. [8]; ${ }^{\mathrm{c}}$ Exp., Ref. [5]

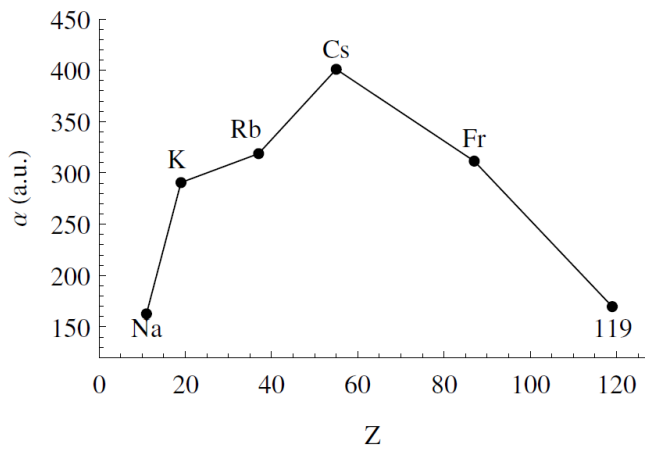

Figure 1. Polarizabilities of group-1 elements

The calculated $\alpha, R_{\max }$, and $\Delta H_{\text {ads }}$ of group- 1 elements are presented in Table I. The obtained $\alpha$ of Cs is in excellent agreement with the experimental value $(401.0 \pm 0.6$ [9]); similar accuracy can be expected from our predictions for Fr and element 119. For the neutral atoms, $\alpha$ (Fig. 1) and $R_{\mathrm{vdW}}$ increase from $\mathrm{Na}$ to $\mathrm{Cs}$ and then decrease towards element 119 , which can be explained by the strong relativistic contraction of the valence $n s$ orbital in the heavier atoms in the group. In fact, the same trend reversal at Cs is also observed for the ionization potentials and electron affinities of group-1 atoms, discussed in Ref. 10. In case of the cations, a different trend in the polarizability is observed, defined by the outer $(n-1) p_{3 / 2}$ orbital, which expands in the group with the increase in the atomic number. Thus, $119^{+}$will have the highest polarizability of group- 1 cations. The $-\Delta H_{\text {ads }}$ in the group decrease with the increase in the atomic number, and the predicted $\Delta H_{\text {ads }}$ of element 119 on Teflon is the lowest among the atoms considered here, as is the case with its $-\Delta H_{\text {ads }}$ on noble metals [10] . The low value of $17.6 \mathrm{~kJ} / \mathrm{mol}$ indicates that this atom should be easily transported through the Teflon capillaries to the chemistry set up.

\section{References}

[1] U. Kaldor, J. Phys. B 6, 71 (1973)

[2] K. Faegri, Theor. Chim. Acta 105, 252 (2001)

[3] DIRAC08, written by H. J. Ja. Jensen et al. (2008)

[4] V. Pershina and T. Bastug, Chem. Phys. 311, 139 (2005)

[5] A. Bondi, J. Phys. Chem. 68, 441 (1964)

[6] J. Desclaux, At. Data Nucl. Data Tables 12, 311 (1973)

[7] V. Holmgen et al., Phys. Rev. A 89053607 (2010)

[8] I.S. Lim et al., J. Chem. Phys. 116, 172 (2002)

[9] J. M. Amini and H. Gould, Phys. Rev. Lett. 91, 153001 (2003)

[10] V. Pershina et al., Chem. Phys. 395, 87 (2012) 